\title{
Comparisons of Behavioral and Neurochemical Characteristics between WKY, WKHA, and Wistar Rat Strains
}

\author{
Guy Drolet, Ph.D., Karine Proulx, B.Sc., Debra Pearson, M.Sc., Joseph Rochford, Ph.D.,
} and Christian F. Deschepper, M.D.

WKHA rats constitute a recombinant inbred rat strain derived by phenotypic selection of the progeny of hybrid F2 crosses between SHR and WKY rats. WKHA are normotensive and show some features of hyperactivity and of hyper-reactivity to stress, but their utility as model of attention deficit/hyperactivity disorder (ADHD) has not yet been settled. To address these questions, we performed behavioral and neurochemical evaluations of WKHA, and compared them to both WKY and Wistar (WIS) rats. In locomotor activity tests, the respective scores for each strain were WKY $<W K H A<W I S$. The relative amplitudes of the inhibition of the startle response by preexposure of the animals to a low-level acoustic cue (prepulse inhibition (PPI) stimulus filtering test) were WKY $<W K H A=W I S$. In nontreated rats, prepulse inhibition was significantly lower in WKY than in WIS and WKHA rats at low prepulse intensity.

Methylphenidate did not decrease locomotor activity in any of the strains, but rather increased locomotion, with the effect being of higher amplitude in WKY. Methylphenidate also impaired prepulse inhibition in a dose-dependent manner. In situ hybridization for NGFI-B, a transcription factor related to the dopaminergic system, revealed that methylphenidate increased the expression of NGFI-B mRNA in the accumbens (core/shell) and striatum only in WKY rats. We conclude that: (1) the behavioral differences between WKY and WKHA can be more readily explained by deficits present in WKY than by unusual characteristics of WKHA, (2) the WKHA does not appear to represent a useful model of $A D H D$, and (3) the use of WKY rats as the only control for behavioral studies is potentially misleading, because they show a combination of behavioral and neurochemical (dopamine-related) characteristics that make them very different from other common rat strains. Although these characteristics may make them interesting models in their own right, it also follows that the conclusions of any study based on comparisons of one particular strain to just WKY should be interpreted very cautiously.

[Neuropsychopharmacology 27:400-409, 2002] (C) 2002 American College of Neuropsychopharmacology. Published by Elsevier Science Inc.
KEY WORDS: Attention deficit; Hyperactivity; NGFI-B; Prepulse inhibition; Methylphenidate.

From the CHUL Research Centre, Neuroscience Unit, Université Laval, Québec, Canada; Douglas Hospital Research Center, Psychiatry Department, McGill University; and Institut de Recherches Cliniques de Montréal, Montréal, Québec, Canada

Address correspondence to: Dr. Guy Drolet, CHUL Research Centre, Neuroscience Unit, 2705 boul. Laurier Sainte-Foy, Québec, Canada G1V 4G2. E-mail: gdrolet@crchul.ulaval.ca

Received August 20, 2001; Revised November 30, 2001; Accepted January 29, 2002.

Online publication: 2/5/02 at www.acnp.org/citations/ Npp020502239.
Wistar-Kyoto-derived Hyperactive (WKHA) rats constitute an inbred strain that has been derived from the progeny of hybrid Wistar-Kyoto (WKY)/ Spontaneously Hypertensive Rats (SHR) F2 crosses. The WKHA rats were developed initially in 1980 by Hendley and associates in the attempt to remove a prominent behavioral abnormality (hyperactivity) from the hypertensive trait of SHR that had been fixed in this strain during intensive inbreeding (Hendley and Ohlsson 1991; Hendley 2000). Using cross-breeding of SHR with WKY followed by recombinant selected inbreeding, Hendley and Ohlsson (1991) developed the inbred WKHT rat 
strain, which is hypertensive but not hyperactive, and the inbred WKHA strain, which is hyperactive and hyperreactive to stress, but not hypertensive. The WKHA are now truly inbred and their genetic makeup constitutes a true mix of the backgrounds of the parental WKY and SHR strains (Deschepper et al. 1997).

WKHA display marked locomotor hyperactivity and hyperreactivity to stress when compared with WKY. The activity score obtained with WKHA in an exploration test in a novel environment is much higher than the score observed in the WKY strain (Sagvolden et al. 1992). Moreover, the WKHA also seems to display "attentional problems" when compared with WKY (Sagvolden et al. 1992). Although these characteristics are reminiscent of those observed in children diagnosed with attention deficit disorder (ADHD), the utility of this strain as a potential model of ADHD has not received extensive experimental attention.

Accordingly, the present study was conducted to assess the face validity of the WKHA strain as a model of ADHD. To do so, we compared the performance of WKHA to both WKY (genetic control) and Wistar (outbred control) strains in two behavioral tests. First, we measured activity levels of the three strains in an exploration test in a novel environment. Second, we examined the behavior of the three strains in the prepulse inhibition (PPI) test of the acoustic startle reflex. This test assesses sensorimotor gating, or the extent to which a weak acoustic stimulus draws cognitive processing away from a more pronounced acoustic stimulus (Ralph et al. 1999; Wood et al. 1998). Although there is considerable debate as to whether PPI reflects the operation of an attentional (as opposed to a preattentional) mechanism, it is clear that PPI is involved in stimulus selection or stimulus filtering, a process that appears to be aberrant in ADHD (Ralph et al. 1999). Moreover, some evidence also exists for a deficit in sensorimotor gating in a sub-population of ADHD children (Castellanos et al. 1996). A third reason justifying our use of the PPI test is that, like locomotor activity, sensorimotor gating is modulated by central dopaminergic substrates (Swerdlow et al. 1994), and there is extensive clinical evidence implicating alterations in dopaminergic function in the pathophysiology of ADHD (Pliszka et al. 1996).

We also examined the extent to which the performances of the three strains in both the locomotor activity and PPI tests were sensitive to pretreatment with the psychomotor stimulant methylphenidate, which is the pharmacologic intervention of choice in the treatment of ADHD. Evidence supporting the face validity of the WKHA strain as a model of ADHD would be provided by the demonstrations that, relative to both WKY and Wistar, WKHA exhibit greater activity counts in the novel environment exploration test and impaired PPI. Additionally, both these behavioral im- pairments should be reversed, at least in part, by pretreatment with methylphenidate.

Finally, in light of possible dopaminergic defects, we examined the expression of NGFI-B, a transcription factor whose expression has been shown to be regulated by dopaminergic tone (Gervais et al. 1999; Beaudry et al. 2000). Expression of this transcription factor was examined within mesocorticolimbic structures that have been proposed to play important roles both in motor activity and attention (Lou et al. 1998; Swanson et al. 1998; Vaidya et al. 1998). NGFI-B expression was examined in both untreated and methylphenidate-treated animals from all three strains.

\section{MATERIALS AND METHODS}

\section{Animals}

WKY/Cr and Wistar [Crl:(WI)BR] were obtained from Charles River (St. Constant, QC, Canada), whereas WKHA/Cfd originated from the colony maintained at the Institut de Recherches Cliniques de Montréal (IRCM) (Deschepper et al. 1997; Masciotra et al. 1999), as registered with the Institute of Laboratory Animal Resources (ILAR) of the National Research Council. The nomenclature of each strain is in compliance with the recommendations of the International Rat Genetic Nomenclature Committee. Rats were used only once and were divided into four pharmacological groups: a vehicle group (saline s.c. $30 \mathrm{~min}$ before the behavioral test) and 3 groups treated with either 1,2, or $5 \mathrm{mg} / \mathrm{kg}$ b.w. of methylphenidate s.c. $30 \mathrm{~min}$ before the behavioral test. The drug was a kind gift of Novartis (Montréal, QC, Canada).

\section{Behavioral Tests}

The three strains described above were used for behavioral tests. All rats were males used at 12 weeks of age. Each animal was used only once, and was tested in either the open field test or the prepulse inhibition test. Ambulation (locomotor activity) in a novel environment was measured under standard room illumination between 10:00 A.M. and 2:00 P.M., using a square Lucite cage $(30 \times 30 \mathrm{~cm})$ equipped with 4 sets of light beams, as used and described previously in the original work of Dr. Hendley for the development of the WKHA strain (Hendley and Ohlsson 1991). The animals were placed in the cage for a total of $10 \mathrm{~min}$, and locomotor activity was measured by calculating the number of times the light beams were interrupted. The experimental cage was cleaned with a $70 \%$ ethanol solution following each subject evaluation.

Prepulse inhibition was measured in two startle chambers (San Diego Instruments, San Diego, CA), consisting each of Plexiglas restrainers mounted on a Plexiglas base within a sound-attenuating chamber. A piezo- 
electric strain meter attached to the base transduced the startle response. Stabilimeter readings were rectified, digitized on a 4095 scale, and recorded by a computer. A speaker located in the ceiling of the sound attenuating chamber presented all acoustic stimuli and maintained a constant background noise level of $70 \mathrm{~dB}$. Startle reactivity was assessed by exposing animals to a 30 msec, $120 \mathrm{~dB}$ acoustic stimulus alone. An average of 50 1-msec readings, beginning at the onset of the startle stimulus, was used as the dependent variable.

PPI of acoustic startle responses was measured by having the $120 \mathrm{~dB}$ startle stimulus preceded by a $30 \mathrm{msec}$ prepulse stimulus, which terminated $70 \mathrm{msec}$ before the onset of the startle stimulus. The intensity of the prepulse stimulus varied from $3-15 \mathrm{~dB}$ above the background noise level in $3 \mathrm{~dB}$ increments. A test session consisted of placing the animals in the startle chamber for a 5-min acclimatization period after which they were exposed to a total of 37 trials separated by variable interstimulus intervals that averaged $15 \mathrm{~s}$. The first two initial trials were startle trials. Results of the very first two trials were discarded as animals generally overreact to them. Over the last 35 trials, animals were exposed to an additional 10-startle trials, and to 5 trials at each of the 5 prepulse intensities. These trials were presented randomly, with the one restriction that no more than two trials of the same type could occur in succession. The Plexiglas restrainers were cleaned with a $70 \%$ ethanol solution between each rat evaluation. For data analysis, the average of the last 10-startle trials was taken as the measure of startle reactivity for each animal. We also averaged the 5 trials taken at each of the 5 prepulse intensities, and then expressed these values as a percentage of the average reactivity for the 10 startle trials, using the formula: $\{$ (startle-prepulse) $/$ startle $\} \times 100$.

\section{In Situ Hybridization Histochemistry}

Histochemical hybridization localization of each transcript was carried out as previously described (Dumont et al. 1999; Mansi et al. 2000). The NGFI-B cRNA probe was generated from a $2.4 \mathrm{~kb}$ EcoR1 fragment of a fulllength rat NGFI-B cDNA (Dr. Milbrandt, Washington University, St. Louis, MO) subcloned into pBluescript SK-1 plasmid that was linearized with BamH I. Radioisotope-labeled antisense cRNA copies were synthesized by incubating 250ng of linearized plasmid in 5X transcription buffer $\left(6 \mathrm{mM} \mathrm{MgCl}_{2} ; 30-40 \mathrm{mM}\right.$ Tris ( $\left.\mathrm{pH} 7.9\right) ; 10 \mathrm{mM}$ $\mathrm{NaCl}), 10 \mathrm{mM}$ dithiothreitol, $0.2 \mathrm{mM}$ ATP/GTP/CTP, $200 \mu \mathrm{Ci}\left\{\alpha-{ }^{35} \mathrm{~S}\right\}$ UTP, 40U RNAsin, and 20U T3 RNA polymerase, together for $60 \mathrm{~min}$ at $37^{\circ} \mathrm{C}$. The RNA-radioprobe was purified with mini-Quick-spin RNA columns (Roche Diagnostics, Montréal, QC, Canada). The cRNA was precipitated with $80 \mu \mathrm{l} 5 \mathrm{M}$ ammonium acetate and $500 \mu \mathrm{l} 100 \%$ ethanol for $20 \mathrm{~min}$ on dry ice. The pellet was resuspended in $100 \mu \mathrm{l} 10 \mathrm{mM}$ Tris/1 mM EDTA ( $\mathrm{pH} 8.0$ ). A concentration of $10^{7} \mathrm{cpm}$ probe was mixed into $1 \mathrm{ml}$ hybridization solution ( $518 \mu \mathrm{l}$ formamide; $62 \mu \mathrm{l} 5 \mathrm{M} \mathrm{NaCl}$; $10 \mu \mathrm{l} 1 \mathrm{M}$ Tris (pH 8.0); $2 \mu \mathrm{l} 0.5 \mathrm{M}$, EDTA (pH 8.0); $20 \mu \mathrm{l} 50 \mathrm{x}$ Denhart's solution; $207 \mu \mathrm{l} 50 \%$ dextran sulfate; $50 \mu 110$ $\mathrm{mg} / \mathrm{ml}$ transfer RNA; $10 \mu \mathrm{l} 1 \mathrm{M}$ dithiothreitol; $118 \mu \mathrm{l}$ diethylpyrocarbonate water-volume of probe used). This solution was mixed and heated for $5 \mathrm{~min}$ at $65^{\circ} \mathrm{C}$ before applying to slides. Radio-isotope-labeled sense (control) cRNA copies were also prepared to verify the specificity of NGFI-B probe. Hybridization with these probes did not reveal any positive signal in the rat brain.

\section{Quantitative Analysis}

Quantification of hybridization signal for the NGFI-B mRNA was performed by analysis of exposed X-ray films (Kodak). Selected regions of interest (ROIs) included the following: parts of the mesocorticolimbic system and of the medial prefrontal cortex, cingular cortex, striatum (divided in dorsomedial and dorsolateral portions), the nucleus accumbens (core and shell), and the ventral tegmental area. Each film was analyzed in a double-blinded fashion. Transmittance values (referred to here as optical density or OD) of the hybridization signal were measured using a Northern Light Desktop Illuminator (Imaging Research, Inc., Ontario) with a Micro-Nikkor 60-mm-mounted Sony Camera coupled to a Power Macintosh 8600/300. The signal was analyzed with NIH image software, version 1.61 (W. Rasband, NIH, Bethesda, MD). Densitometric analysis, yielding measures of integrated OD (area of the signal $\times$ average optical density), were performed according to a standard scale established using ${ }^{14} \mathrm{C}$ standard slides (American Radiolabeled Chemicals Inc., St. Louis, MO). All samples that emitted a clear positive signal were evaluated within a linear range to avoid pixel saturation and underestimation. The OD of each side of ROI was corrected for the average background signal by subtracting the OD of areas without positive signal located immediately outside the digitalized ROI.

\section{Statistical Analysis}

Locomotor activity data were analyzed by a betweensubjects, two-way (strain $\times$ drug treatment) ANOVA, followed by Fisher LSD post-hoc tests. PPI data in nontreated animals were analyzed by two-way factorial ANOVA (strain $\times$ prepulse intensity, with prepulse intensity as a repeated measures factor), followed by Tukey's post-hoc test. PPI data in methylphenidatetreated animals were analyzed by a three-way factorial ANOVA (strain $\times$ methylphenidate dose $\times$ prepulse intensity, with prepulse intensity as a repeated measures factor), followed by Tukey's post-hoc test. In situ hybridization data were expressed as relative total integrated density (arbitrary units) for NGFI-B mRNA of the ROI of control (vehicle) or methylphenidate rats. 


\section{LOCOMOTOR ACTIVITY}

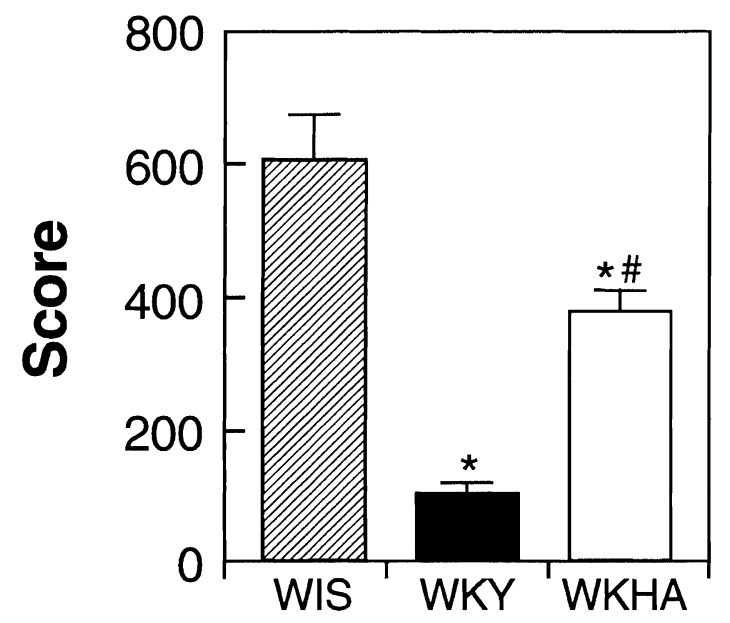

B METHYLPHENIDATE-INDUCED CHANGES IN LOCOMOTION
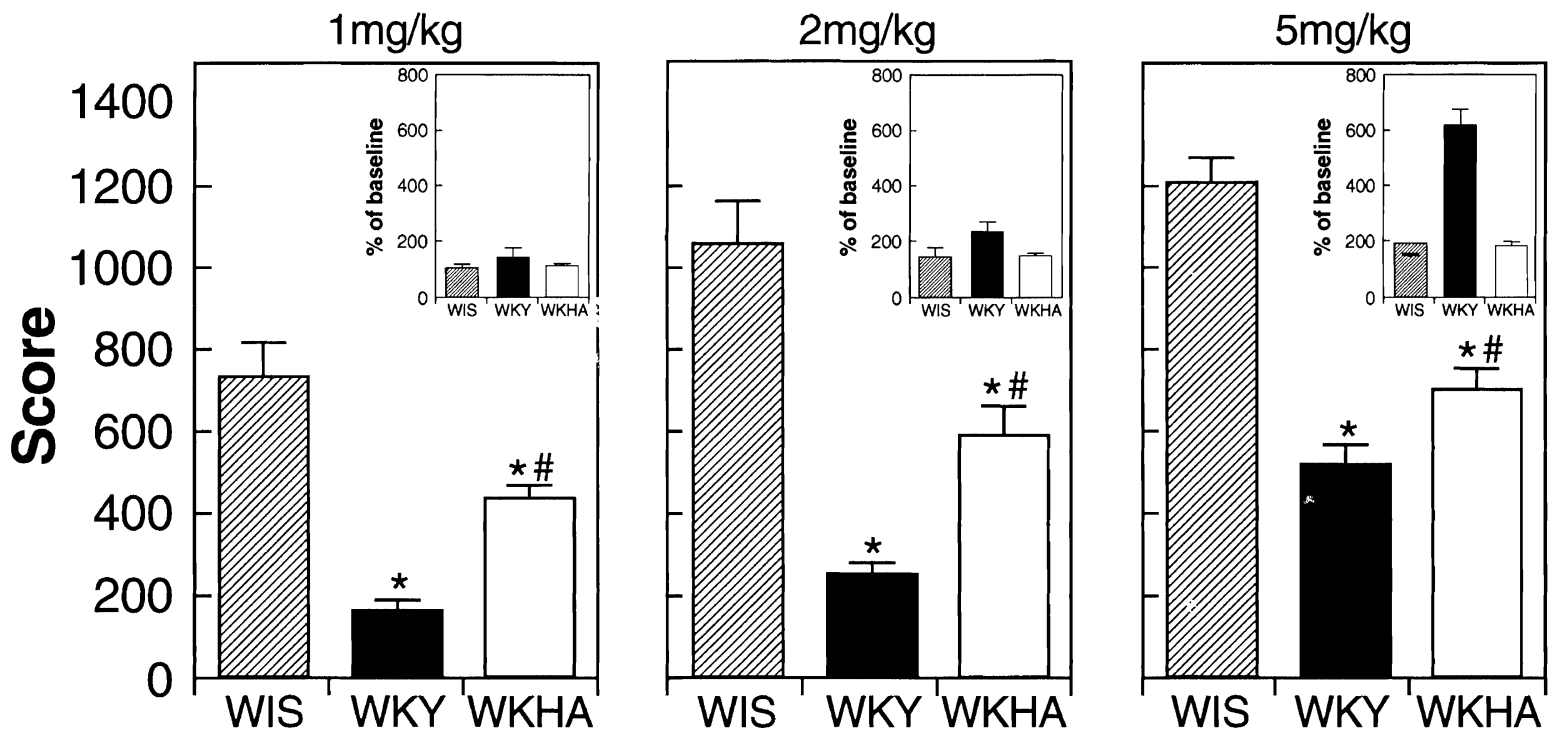

Figure 1. Mean $( \pm$ SEM) activity scores in Wistar, WKY, and WKHA male rats treated with either saline (panel A) or 1, 2, or 5 $\mathrm{mg} / \mathrm{kg}$ methylphenidate (panels in B) prior to exposure to a novel open field. Each bar in each panel represents the results from a separate group of animals ( $n=6-8$ per group). The insets in (B) represent the percentage change for each dose compared with the respective saline control groups. Significant differences are indicated as ${ }^{*} p<.01 \mathrm{vs}$. WIS and \# $p<.01 \mathrm{vs.} \mathrm{WKY.}$

The results were analyzed using a two-way betweensubject factorial ANOVA (strain $\times$ drug treatment); post-hoc multiple comparisons were done using a Bonferroni test. Levels were deemed significant at $p<.05$.

\section{RESULTS}

\section{Exploration Activity in a Novel Environment}

Figure 1A depicts the mean scores of locomotor activity for the three strains. The strain effect was character- ized by greater activity in WIS than WKHA $(p<.0001)$, which were more active than the WKY $(p<.0001)$ (Figure $1 \mathrm{~A})$. Methylphenidate administration $(1,2$, and 5 $\mathrm{mg} / \mathrm{kg} \mathrm{b.w.)} \mathrm{induced} \mathrm{a} \mathrm{dose-dependent} \mathrm{increase} \mathrm{in} \mathrm{lo-}$ comotor activity in the three strains (Figure 1B). The percentage increase of locomotion to methylphenidate, however, was much higher in WKY, especially with the $5 \mathrm{mg} / \mathrm{kg}$ dose, whereas the percentage increase for WKHA and WIS were to the same extent (Figure 1B, inset). 


\section{PREPULSE INHIBITION}

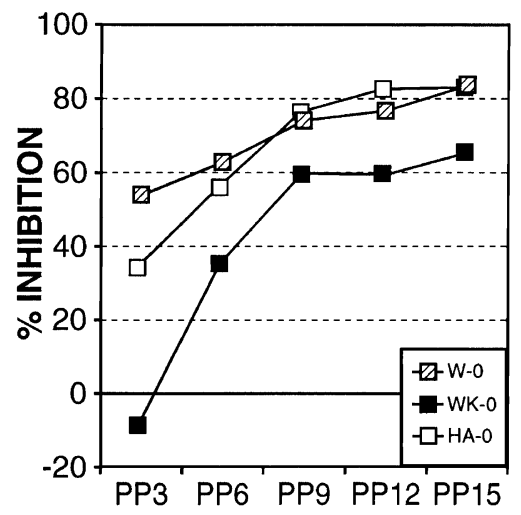

\section{METHYLPHENIDATE-INDUCED CHANGES IN PREPULSE INHIBITION}
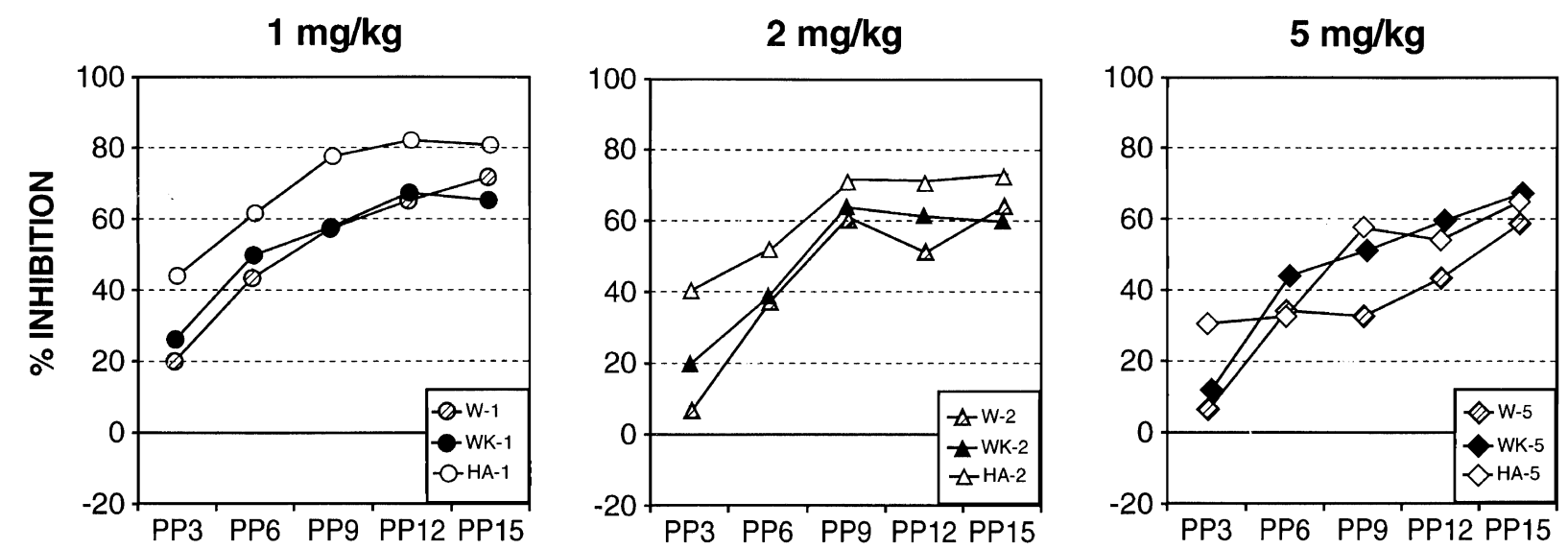

Figure 2. Mean $( \pm \mathrm{SEM})$ prepulse inhibition in Wistar, WKY, and WKHA male rats administered either saline (panel A) or 1,2 , or $5 \mathrm{mg} / \mathrm{kg}$ methylphenidate (panels in B) across 5 different levels of prepulse intensities. Each function in each panel represents the results from a separate group of animals $(n=6$ per group). A three-way ANOVA revealed significant main effects for strain $(p<.025)$, indicating lowered PPI in Wistar animals relative to WKHA, drug treatment $(p<.03)$, indicating a dose-dependent reduction in PPI, and prepulse intensity $(p<.0001)$, indicating increasing levels of PPI across increasing prepulse intensities. There were no significant interactions.

\section{Prepulse Inhibition}

Figure 2A shows the percent of prepulse inhibition exhibited by the three strains at each of the five intensities in saline-treated animals. ANOVA yielded significant main effects for strain $\left(\mathrm{F}_{(2,15)}=7.63, p<.01\right)$, for prepulse intensity, $\left(\mathrm{F}_{(4,60)}=41.53, p<.0001\right)$, and a significant interaction between both factors, $\left(\mathrm{F}_{(8,60)}=3.21, p<.005\right)$. The prepulse intensity main effect reflects the increase in the magnitude of PPI with increases in prepulse intensity. Tukey's analysis of the interaction revealed that prepulse inhibition was significantly lower in WKY than in WIS and WKHA rats at PP3, and significantly lower than WIS at PP6 $(p<.05)$.
Figure $2 \mathrm{~B}$ shows the percent of prepulse inhibition exhibited by the 3 strains at each of the 5 intensities in animals treated with 3 different doses of methylphenidate. ANOVA yielded significant main effects for strain, $\left(\mathrm{F}_{(2,45)}=3.89, p<.03\right)$, dose, $\left(\mathrm{F}_{(2,45)}=3.356, p<.05\right)$, and prepulse intensity, $\left(\mathrm{F}_{(4,180)}=81.25, p<.0001\right)$. None of the interactions were significant. The prepulse intensity effect reflects the increase in PPI with increases in prepulse intensity. The strain main effect reflects the finding that WKHA rats exhibited significantly greater $(p<.05)$ levels of PPI in comparison to WIS (but not WKY). The dose main effect indicates that administration of $5 \mathrm{mg} / \mathrm{kg}$ methylphenidate significantly $(p<.05)$ 


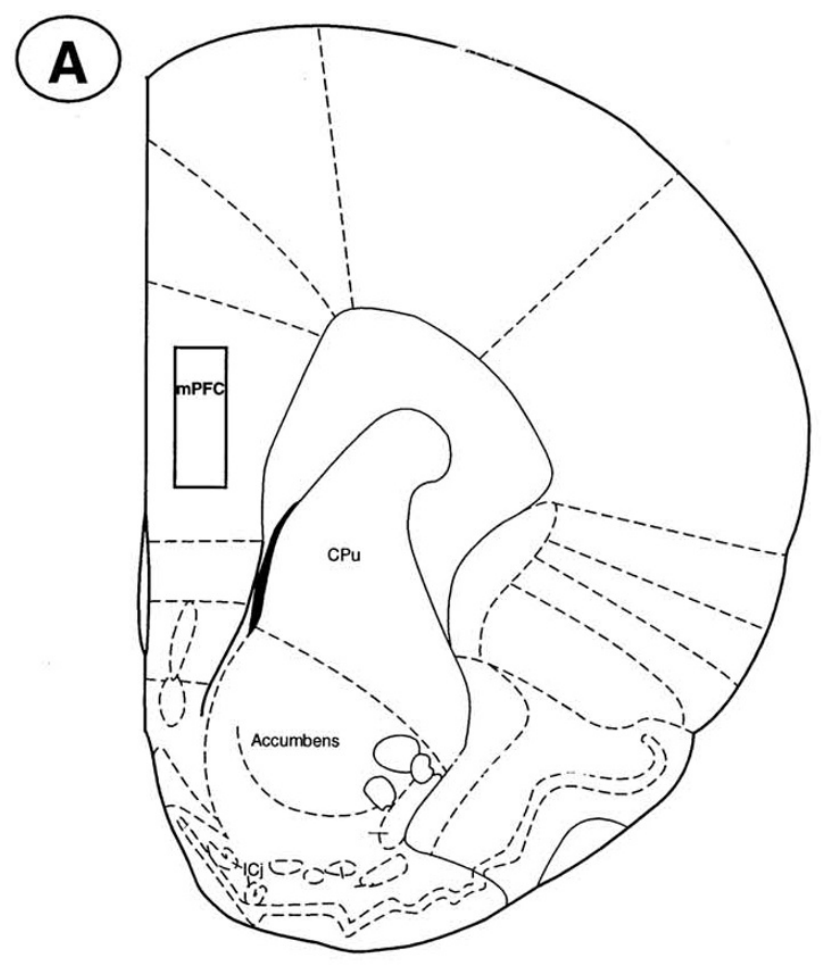

(B)
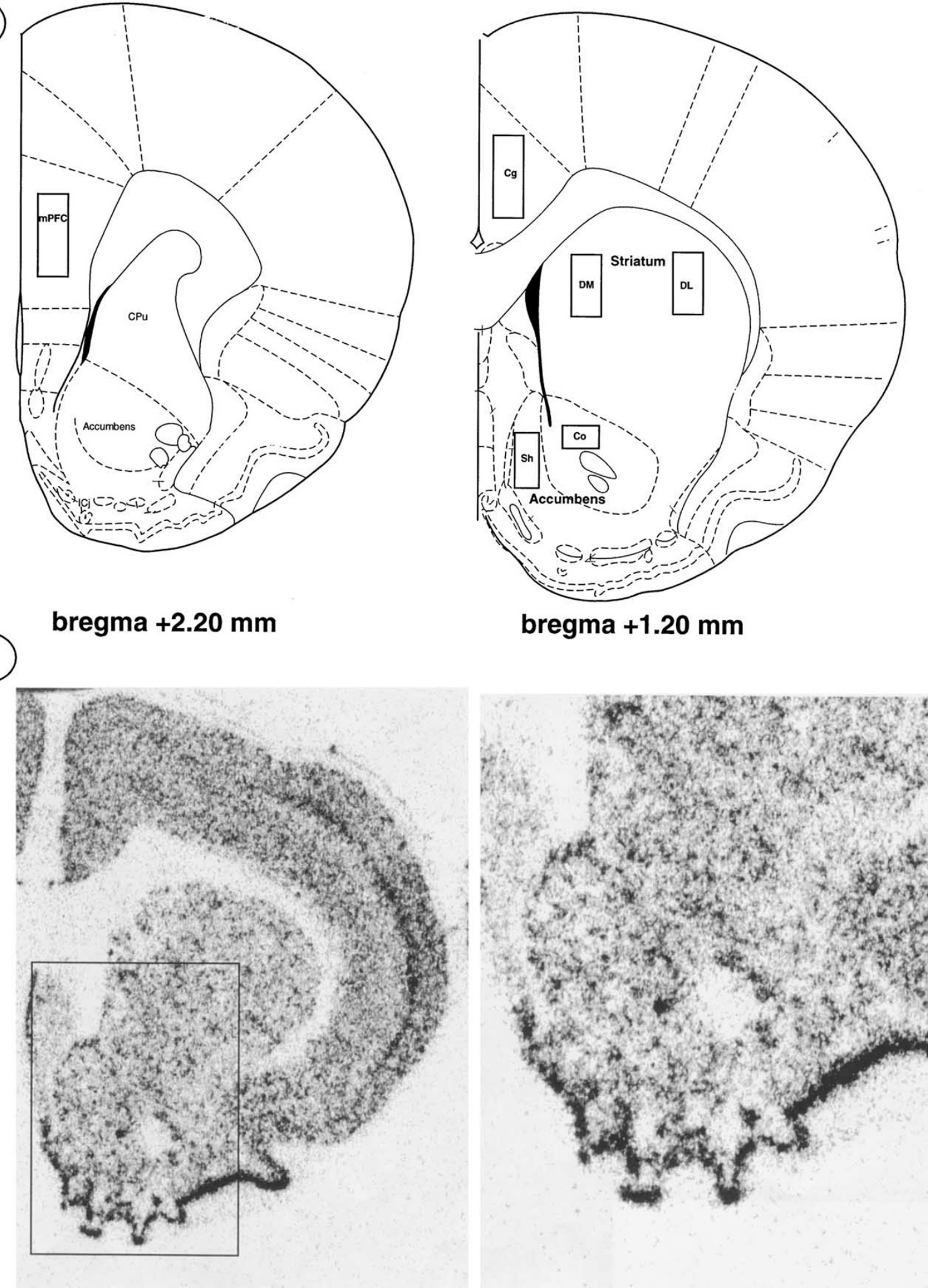
reduced levels of PPI relative to the $1 \mathrm{mg} / \mathrm{kg}$ (but not the $2 \mathrm{mg} / \mathrm{kg}$ ) dose.

\section{NGFI-B Expression}

We examined the expression of NGFI-B (a transcription factor whose expression is regulated by dopaminergic tone) within mesocorticolimbic structures (Figure 3). In the accumbens, ANOVA yielded a significant interaction between strain and methylphenidate treatment both within the core $\left(\mathrm{F}_{(2,60)}=4.39, p<.017\right)$ and the shell $\left(\mathrm{F}_{(2,61)}=4.18, p<.02\right)$ divisions. In the striatum, ANOVA yielded significant main effects for strain $\left(\mathrm{F}_{(2,61)}=4.05, p<.022\right)$, for methylphenidate treatment $\left(\mathrm{F}_{(1,61)}=5.61, p<.021\right)$ and a significant interaction between strain and methylphenidate treatment $\left(\mathrm{F}_{(2,61)}=\right.$ $5.32, p<.0074)$. In the cingular cortex, ANOVA yielded a significant interaction between strain and methylphenidate treatment $\left(\mathrm{F}_{(2,61)}=3.89, p<.03\right)$. Finally, in the medial prefrontal cortex ANOVA yielded a significant interaction between strain and methylphenidate treatment $\left(\mathrm{F}_{(2,32)}=0.70, p>.5\right)$. There was no difference in basal levels in the NGFI-B expression between the three rat strains for any of the structures investigated, i.e., striatum, accumbens (core and shell), medial prefrontal cortex, and cingular cortex (Figure 4). Methylphenidate treatment $(5 \mathrm{mg} / \mathrm{kg})$ induced a differential pattern of response in each rat strain. In Wistar and WKHA, methylphenidate had only marginal effects. Thus, the only noticeable effect in Wistar was a trend toward a decrease $(p<.06)$ in the core of the nucleus accumbens. In WKHA, the only significant effect of methylphenidate was a small increase of NGFI-B mRNA expression within the shell of the accumbens. In contrast to the two other strains, methylphenidate significantly increased the expression of NGFI-B mRNA in the accumbens (core and shell), striatum, and cingular cortex of WKY (Figure 4).

\section{DISCUSSION}

In order to assess the validity of the WKHA strain as an animal model of ADHD, the present experiments contrasted the locomotor and sensorimotor gating capacities of this strain with those of the WKY and Wistar strains, and also examined the effects of methylpheni- date administration to each strain in each behavioral test. As indicated previously, evidence supporting the face validity of the model would be provided by the findings that (1) WKHA animals show deficits in PPI, (2) are hyperactive in the open field test, and (3) both these behavioral impairments are reversed by methylphenidate administration.

The results obtained in saline-treated animals failed to confirm the first two predictions. PPI in saline-treated WKHA rats was no different from that in saline-treated WIS rats. Moreover, the strain that appeared to be most impaired in terms of PPI performance was WKY, as PPI in WKY animals was lower than that in WKHA and WIS, at least at the low levels of prepulse intensity. In the open field test, saline-treated WKHA rats were indeed more active in the open field exploration test in comparison with the inbred control WKY strain, as has been previously reported (Sagvolden et al. 1992). The activity of WKHA rats was, however, lower than that observed in the outbred Wistar control strain and also that of Lewis rats (results not shown). Several studies have reported that the WKY strain displays marked hypoactivity in open field tests (Tilson et al. 1977; McCarty 1983; Pare and Kluczynski 1997). Accordingly, it appears that the difference in locomotor activity between WKHA and WKY is the result of hypoactivity in WKY, rather than the putative hyperactivity of WKHA.

Because methylphenidate has been shown to ameliorate both the hyperactivity and the attention deficits observed in ADHD, we also characterized the effects of this compound in the three strains in both the open field and the PPI tests. In both cases, methylphenidate failed to evoke the predicted outcome, a reduction of locomotion and an increase in prepulse inhibition. In the open field, methylphenidate dose-dependently increased locomotor activity in all strains. Moreover, of the three strains examined, the response of WKY to methylphenidate administration was the most pronounced. Specifically, at the $5 \mathrm{mg} / \mathrm{kg}$ dose, methylphenidate increased locomotor activity approximately six-fold in WKY, but only by about two-fold in both WKHA and Wistar.

WKHA rats administered methylphenidate displayed significantly higher levels of PPI than did Wistar rats treated with this compound. This could, in principle, be taken as partial support for the hypothesis that methylphenidate improves PPI performance in the

\footnotetext{
Figure 3. Schematic illustration of rat forebrain regions analyzed for NGFI-B mRNA expression (A). These levels correspond to bregma +1.20 and +2.20 of the Paxinos and Watson Atlas. Specific brain areas sampled for mRNA analysis are shown in rectangular boxes. (B) Representative autoradiograms of the same coronal section, at different magnification, depicting level of expression for NGFI-B mRNA in the nucleus accumbens of vehicle-treated Wistar rat. These photos were taken from autoradiogram of 30- $\mu \mathrm{m}$ coronal sections hybridized with ${ }^{35} \mathrm{~S}$ NGFI-B riboprobe through corresponding sections of the nucleus accumbens. Cingular cortex $(\mathrm{Cg})$, shell (Sh), and core (Co) of the accumbens, medial prefrontal cortex (mPFC), dorsolateral (DL) and dorsomedian (DM) striatum, CPu caudate-putamen.
} 
NGFI-B mRNA Expression
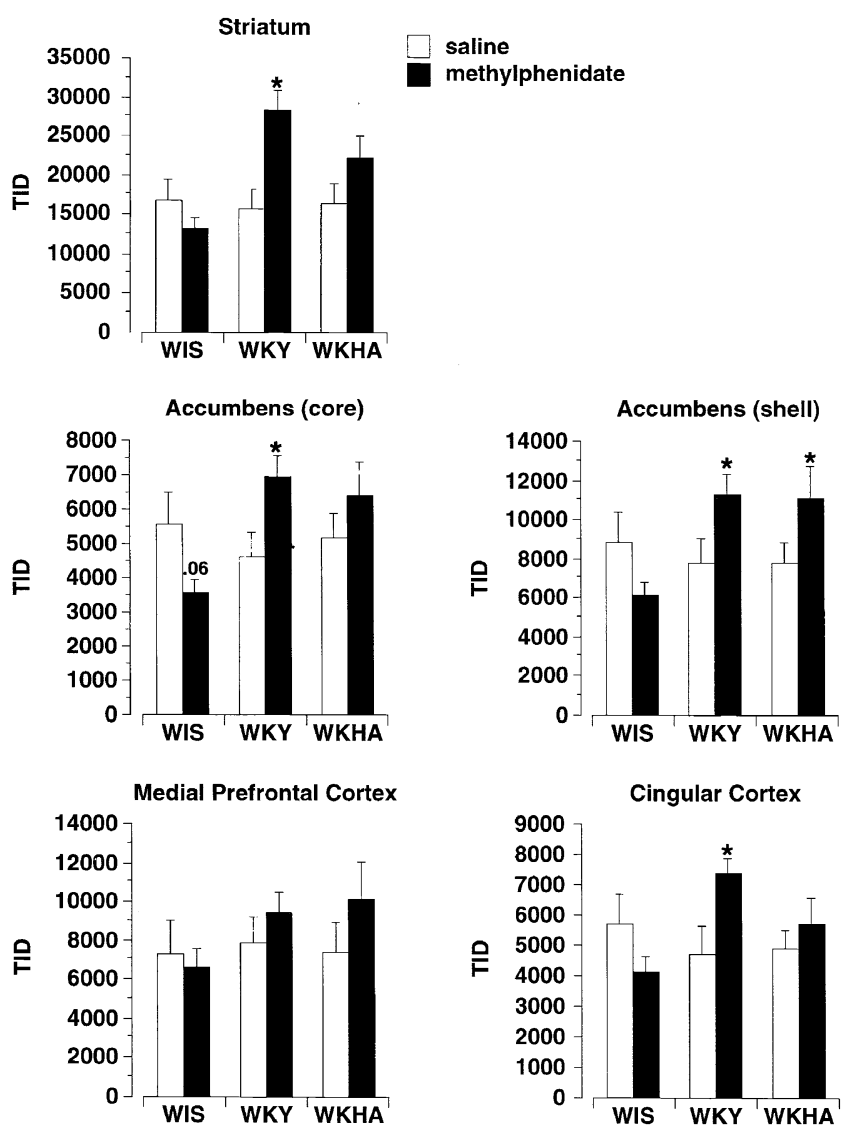

Figure 4. Effects of methylphenidate administration (5 $\mathrm{mg} / \mathrm{kg}$ ) on NGFI-B mRNA expression in the striatum, accumbens, medial prefrontal cortex and cingular cortex in Wistar, WKY, and WKHA male rats. ( $n=6$ per group). * indicates a significant difference $(p<.05)$ as compared with the saline group. TID (total integrated density = optical density in $\mu \mathrm{Ci} / \mathrm{gr} \times$ area in square pixel).

WKHA strain. There are, however, two problems with this conclusion. First, PPI levels in methylphenidatetreated WKHA rats were no different from those in methylphenidate-treated WKY animals. Second, and more importantly, within-strain comparisons of the effects of methylphenidate (Figures 3A and 3B) suggest that this compound actually impaired PPI performance in Wistar animals, rather than improve performance in the WKHA rats. For instance, at the highest dose administered $(5 \mathrm{mg} / \mathrm{kg})$ methylphenidate reduced PPI levels in WKHA rats by about $20-30 \%$ across the different prepulse intensities relative to untreated WKHA rats. In Wistar animals, $5 \mathrm{mg} / \mathrm{kg}$ methylphenidate evoked a more pronounced impairment that ranged from $30-50 \%$.

The finding that methylphenidate impaired PPI is not particularly surprising, in that administration of other psychostimulants (e.g., amphetamine) also disrupt PPI (Kinney et al. 1999; Lacroix et al. 1999; Sills 1999). What is perhaps more noteworthy is the finding that it did so in a strain-dependent manner (Kinney et al. 1999). Further evidence for this conclusion is provided by the observation that methylphenidate evoked a qualitatively different response on PPI in the WKY strain; $5 \mathrm{mg} / \mathrm{kg}$ evoked a $314 \%$ increase in PPI at the PP3 intensity, and changes that ranged between $0-15 \%$ at the higher intensities.

Finally, in order to assess dopaminergic tone with a biochemical marker, we examined NGFI-B mRNA levels in both untreated and methylphenidate-treated animals in all three strains. We found that methylphenidate increased NGFI-B mRNA in the accumbens and striatum only in the WKY strain. Recent anatomical evidence suggests a close relationship between NGFI-B and the dopaminergic system (Xiao et al. 1996; Zetterstrom et al. 1996; Gervais et al. 1999). Moreover, NGFI-B gene expression has been shown to be modulated by drugs interacting with dopamine. For instance, quinpirole (a D2 receptor agonist) administered alone induced a significant decrease in striatal NGFI-B mRNA expression (Gervais et al. 1999). Conversely, an acute or chronic treatment with haloperidol (a nonspecific D2, D3, D4 receptor antagonist) induces high NGFI-B mRNA levels in the prefrontal and cingulate cortices, in the nucleus accumbens shell, and in the dorsolateral striatum (Beaudry et al. 2000). In some ways, methylphenidate, amphetamine, and cocaine could be considered as indirect agonists of the dopaminergic system. They all interact with the dopamine transporter to either promote dopamine release or block dopamine uptake which enhances dopamine action at the level of the dopaminergic synapse (Kuczenski and Segal 1997). Therefore, it may seem paradoxical to see that methylphenidate induced an increase in NGFI-B expression in the WKY rather than a decrease as observed with dopamine agonist (Gervais et al. 1999). This is consistent, however, with a recent study reporting that acute or chronic cocaine administration also upregulated the level of NGFI-B mRNA in striatal regions (caudate putamen, accumbens) and cortex cinguli (Werme et al. 2000a). An increase in NGFI-B expression might reflect either a neuronal activation (cellular immediate-early gene, Mansi et al. 1998) or a specific increase in sensitivity for retinoid signaling in these regions (Werme et al. 2000b; Langlois et al. 2001). Whether the increase in NGFI-B expression observed in treated WKY is related either to increased locomotion in response to methylphenidate in this strain or a stress-mediated effect remains to be determined.

Altogether, our behavioral and biochemical data did not provide evidence that WKHA might be a suitable model of ADHD. As a corollary, we found that WKY rats appear to have characteristics on their own that make them different from many other strains. In support to this statement, a recent study has evaluated in SHR, WKY, and Sprague-Dawley (SD) strains the ac- 
quisition and performance in the differential reinforcement of low rate (DRL) paradigm, a test regarded as a behavioral screen from impulsive behavior and a characteristic trait of ADHD (Bull et al. 2000). Bull et al. showed that the SHR did not exhibit impaired acquisition of the DRL task, and that both SHR and SD learned the task significantly quicker than the WKY strain. Interestingly, others have tagged the WKY rats as a possibly stress-sensitive strain (Pare and Redei 1993; Pare and Kluczynski 1997). Numerous reports have indicated that the WKY rats display high anxiety and low locomotion reactivity compared with other strains (Pare 1992a, b, 1993, 1994; Dugovic et al. 2000). Likewise, the WKY have been proposed as an animal model of depressive behavior because they consistently demonstrate exaggerated behavioral and physiologic responses to stress in a variety of situations when compared with other strains (Pare 1993, 1994). Indeed, the WKY strain demonstrates higher levels of behavioral immobility at baseline in the forced swimming test (a rodent test sensitive to antidepressant drugs) and in open field (Pare 1994; Lopez-Rubalcava and Lucki 2000). Thus, these rats possess a combination of behavioral and neurochemical (dopamine-related) characteristics that make them interesting models in their own right. These unusual characteristics, however, may make them unsuitable as "controls" for some behavioral or neurochemical studies. Consequently, the conclusions of any study based on comparisons of one particular strain to just WKY should be interpreted very cautiously.

\section{ACKNOWLEDGMENTS}

This research was supported by grants from the Canadian Institutes for Health Research (CIHR) to GD, JR, and CFD. GD and JR held scholarships from le Fonds de la Recherche en Santé du Québec (FRSQ). DP held a student fellowship from the Natural Sciences and Engineering Research Council of Canada (NSERC). We thank Dr. Milbrandt (Washington University, St. Louis, MO) for the generous gift of the rat NGFI-B cDNA.

\section{REFERENCES}

Beaudry G, Langlois MC, Weppe I, Rouillard C, Levesque D (2000): Contrasting patterns and cellular specificity of transcriptional regulation of the nuclear receptor nerve growth factor-inducible $\mathrm{B}$ by haloperidol and clozapine in the rat forebrain. J Neurochem 75:1694-1702

Bull E, Reavill C, Hagan JJ, Overend P, Jones DN (2000): Evaluation of the spontaneously hypertensive rat as a model of attention deficit hyperactivity disorder: Acquisition and performance of the DRL-60s test. Behav Brain Res 109:27-35

Castellanos FX, Fine EJ, Kaysen D, Marsh WL, Rapoport JL,
Hallett M (1996): Sensorimotor gating in boys with Tourette's syndrome and ADHD: Preliminary results. Biol Psychiatry 39:33-41

Deschepper CF, Prescott G, Hendley ED, Reudelhuber TL (1997): Genetic characterization of novel strains of rats derived from crosses between Wistar-Kyoto and spontaneously hypertensive rats, and comparisons with their parental strains. Lab Anim Sci 47:638-646

Dugovic C, Solberg LC, Redei E, Van Reeth O, Turek FW (2000): Sleep in the Wistar-Kyoto rat, a putative genetic animal model for depression. Neuroreport 11:627-631

Dumont EC, Rafrafi S, Laforest S, Drolet G (1999): Involvement of central angiotensin receptors in stress adaptation. Neuroscience 93:877-884

Gervais J, Soghomonian JJ, Richard D, Rouillard C (1999): Dopamine and serotonin interactions in the modulation of the expression of the immediate-early transcription factor, nerve growth factor-inducible $B$, in the striatum. Neuroscience 91:1045-1054

Hendley ED (2000): WKHA rats with genetic hyperactivity and hyperreactivity to stress: A review. Neurosci Biobehav Rev 24:41-44

Hendley ED, Ohlsson WG (1991): Two new inbred rat strains derived from SHR: WKHA, hyperactive, and WKHT, hypertensive, rats. Am J Physiol 261:H583-H589

Kinney GG, Wilkinson LO, Saywell KL, Tricklebank MD (1999): Rat strain differences in the ability to disrupt sensorimotor gating are limited to the dopaminergic system, specific to prepulse inhibition, and unrelated to changes in startle amplitude or nucleus accumbens dopamine receptor sensitivity. J Neurosci 19:5644-5653

Kuczenski R, Segal DS (1997): Effects of methylphenidate on extracellular dopamine, serotonin, and norepinephrine: Comparison with amphetamine. J Neurochem 68:20322037

Lacroix L, Broersen LM, Feldon J, Weiner I (1999): Effects of local infusions of dopaminergic drugs into the medial prefrontal cortex of rats on latent inhibition, prepulse inhibition and amphetamine induced activity. Behav Brain Res 107:111-121

Langlois M, Beaudry G, Zekki H, Rouillard C, Levesque D (2001): Impact of antipsychotic drug administration on the expression of nuclear receptors in the neocortex and striatum of the rat brain. Neuroscience 106:117-128

Lopez-Rubalcava C, Lucki I (2000): Strain differences in the behavioral effects of antidepressant drugs in the rat forced swimming test. Neuropsychopharmacology 22:191-199

Lou HC, Andresen J, Steinberg B, McLaughlin T, Friberg L (1998): The striatum in a putative cerebral network activated by verbal awareness in normals and in ADHD children. Eur J Neurol 5:67-74

Mansi JA, Rivest S, Drolet G (1998): Effect of immobilization stress on transcriptional activity of inducible immediate-early genes, corticotropin-releasing factor, its type 1 receptor, and enkephalin in the hypothalamus of borderline hypertensive rats. J Neurochem 70:1556-1566

Mansi JA, Laforest S, Drolet G (2000): Effect of stress exposure on the activation pattern of enkephalin- containing perikarya in the rat ventral medulla. J Neurochem 74:2568-2575 
Masciotra S, Picard S, Deschepper CF (1999): Cosegregation analysis in genetic crosses suggests a protective role for atrial natriuretic factor against ventricular hypertrophy. Circ Res 84:1453-1458

McCarty R (1983): Stress, behavior and experimental hypertension. Neurosci Biobehav Rev 7:493-502

Pare WP (1992a): The performance of WKY rats on three tests of emotional behavior. Physiol Behav 51:1051-1056

Pare WP (1992b): Learning behavior, escape behavior, and depression in an ulcer susceptible rat strain. Integr Physiol Behav Sci 27:130-141

Pare WP (1993): Passive-avoidance behavior in WistarKyoto (WKY), Wistar, and Fischer-344 rats. Physiol Behav 54:845-852

Pare WP (1994): Open field, learned helplessness, conditioned defensive burying, and forced-swim tests in WKY rats. Physiol Behav 55:433-439

Pare WP, Redei E (1993): Depressive behavior and stress ulcer in Wistar Kyoto rats. J Physiol (Paris) 87:229-238

Pare WP, Kluczynski J (1997): Differences in the stress response of Wistar-Kyoto (WKY) rats from different vendors. Physiol Behav 62:643-648

Pliszka SR, McCracken JT, Maas JW (1996): Catecholamines in attention-deficit hyperactivity disorder: Current perspectives. J Am Acad Child Adolesc Psychiatry 35:264272

Ralph RJ, Varty GB, Kelly MA, Wang YM, Caron MG, Rubinstein M, Grandy DK, Low MJ, Geyer MA (1999): The dopamine D2, but not D3 or D4, receptor subtype is essential for the disruption of prepulse inhibition produced by amphetamine in mice. J Neurosci 19:4627-4633

Sagvolden T, Hendley ED, Knardahl S (1992): Behavior of hypertensive and hyperactive rat strains: Hyperactivity is not unitarily determined. Physiol Behav 52:49-57

Sills TL (1999): Amphetamine dose dependently disrupts prepulse inhibition of the acoustic startle response in rats within a narrow time window. Brain Res Bull 48: $445-448$
Swanson J, Castellanos FX, Murias M, LaHoste G, Kennedy J (1998): Cognitive neuroscience of attention deficit hyperactivity disorder and hyperkinetic disorder. Curr Opin Neurobiol 8:263-271

Swerdlow NR, Braff DL, Taaid N, Geyer MA (1994): Assessing the validity of an animal model of deficient sensorimotor gating in schizophrenic patients. Arch Gen Psychiatry 51:139-154

Tilson HA, Chamberlain JH, Gylys JA, Buyniski JP (1977): Behavioral suppressant effects of clonidine in strains of normotensive and hypertensive rats. Eur J Pharmacol 43:99-105

Vaidya CJ, Austin G, Kirkorian G, Ridlehuber HW, Desmond JE, Glover GH, Gabrieli JD (1998): Selective effects of methylphenidate in attention deficit hyperactivity disorder: A functional magnetic resonance study. Proc Natl Acad Sci USA 95:14494-14499

Werme M, Olson L, Brene S (2000a): NGFI-B and nor1 mRNAs are upregulated in brain reward pathways by drugs of abuse: Different effects in Fischer and Lewis rats. Mol Brain Res 76:18-24

Werme M, Ringholm A, Olson L, Brene S (2000b): Differential patterns of induction of NGFI-B, Nor1 and c-fos mRNAs in striatal subregions by haloperidol and clozapine. Brain Res 863:112-119

Wood GK, Tomasiewicz H, Rutishauser U, Magnuson T, Quirion R, Rochford J, Srivastava LK (1998): NCAM-180 knockout mice display increased lateral ventricle size and reduced prepulse inhibition of startle. Neuroreport 9:461-466

Xiao Q, Castillo SO, Nikodem VM (1996): Distribution of messenger RNAs for the orphan nuclear receptors Nurr1 and Nur77 (NGFI-B) in adult rat brain using in situ hybridization. Neuroscience 75:221-230

Zetterstrom RH, Williams R, Perlmann T, Olson L (1996): Cellular expression of the immediate early transcription factors Nurr1 and NGFI-B suggests a gene regulatory role in several brain regions including the nigrostriatal dopamine system. Brain Res Mol Brain Res 41:111-120 